\title{
Two Simple Models of Almost the Same Population with Very Different Dynamics
}

\author{
MAREK KIMMEL \\ Department of Statistics, Rice University, Houston Texas 77251 \\ AND \\ OVIDE ARINO \\ Department of Mathematics, University of Pau, 64000 Pau, France \\ Received 26 May, 1993; revised 11 November 1993
}

\begin{abstract}
We present our point of view of the controversy regarding complex dynamics of population models. We analyze two nonlinear models of population dynamics which may be used to model the same population, but which display very different dynamics. We prove that Model 1 stays globally asymptotically stable, while Model 2 is known to display instability, oscillations, and apparent period doubting leading to chaos. We also indicate situations in which both models are equivalent. We employ these results to argue that the "complex" behavior of some models may be due to apparently small differences in assumptions.
\end{abstract}

\section{INTRODUCTION}

In recent years there was a lot of interest in nonlinear models of population dynamics describing various regulatory phenomena leading to self-limiting growth. Examples of applications include ecology, demography, and cell biology and are not limited to these disciplines [2].

Modeling of population dynamics is based on various principles. Predominantly, the models are based on ordinary or partial differential equations (PDE) or branching processes and more general stochastic population processes. It is not quite clear to what extent these approaches are equivalent in the sense of leading to similar dynamics of models meant to describe similar nonlinear mechanisms.

Regarding mathematical analysis of dynamics of the population models, much attention has been paid recently to "interesting" and "complex" phenomena like bifurcation, oscillations, and chaos, in addition to

(C)Elsevier Science Inc., 1994 
"straightforward" properties like asymptotic stability. Following the same trends, new explanations based on chaos has been suggested for phenomena which traditionally were explained in the terms of stochastic perturbations.

In the present contribution, we relate our own experience with modeling dynamics of populations to these recent trends. We are interested in two general questions. First, when are models derived from different principles equivalent? Second, to what extent is the complex behavior of some models due to their assumptions?

We selected for analysis two nonlinear models of population dynamics which may be used to model the same population, but which display very different dynamics. For simplicity, the models are chosen to describe cell population dynamics (see Discussion), but the conclusions seem to be more general.

We provide an original proof that Model 1 stays globally asymptotically stable (Proposition 4), while Model 2 is known to display instability, oscillations, and apparent period doubling leading to chaos (Proposition 5). Interestingly, there exist situations in which these two models lead to the same equation (Appendices B and C), and this fact may lead to additional confusion since in general they are very different.

\section{THE TWO MODELS}

The following cell population model published by Kimmel [10] is the basis for our analysis (Figure 1):

1. The interdivision times of cells are independent random variables with common distribution density $g(\tau)$.

2. A cell dividing at time $t$ produces a random number $\mu(t)$ of surviving progeny cells $(\mu(t)=1,2$, or 3 ), independent of the number of progeny produced by all other cells and independent of the lifetimes of all cells. The average is $E \mu(t)=m(t)$ surviving progeny per cell.

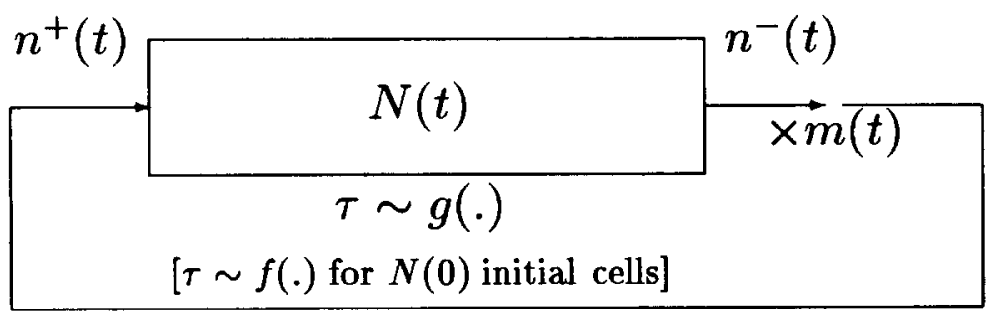

FIG. 1. Schematic diagram of the basic cell population model. 
3. The population is started by $N(0)$ "initial" cells. Each of these cells has a random time left before it can divide. These random times are independent and identically distributed with density $f(\tau)$.

HYPOTHESIS 1

The densities $g$ and $f$ and function $m$ are nonnegative bounded measurable functions on $\mathbf{R}_{+}$such that $\int_{\mathbf{R}_{+}} g=\int_{\mathbf{R}_{+}} f=1$ and $m(t) \leqslant 2$.

Let us denote $N(t)$ the expected cell count at time $t, n^{+}(t)$ the expccted flux of "just born" progeny cells, and $n^{-}(t)$ the expected flux of "just dividing" parent cells. The act of division is assumed to be of zero duration. The two fluxes can be understood as the time derivatives, of $N^{+}(t)$ the cumulated expected count up to time $t$ of progeny cells and of $N^{-}(t)$ the cumulated expected count up to time $t$ of dividing parent cells, respectively. The following equations are then satisfied [10]:

$$
\begin{gathered}
n^{-}(t)=\int_{0}^{t} n^{+}(t-u) g(u) d u+N(0) f(t), \\
n^{+}(t)=m(t) n^{-}(t), \\
N(t)=N(0)+\int_{0}^{t}\left[n^{+}(\tau)-n^{-}(\tau)\right] d \tau, \quad t \geqslant 0 .
\end{gathered}
$$

The next hypothesis makes reformulating the equations possible. HYPOTHESIS 2

The densities $g$ and f have supports restricted to the interval $[0,1]$.

We then have [10]

$$
\begin{gathered}
n^{+}(t)=m(t) \int_{0}^{1} n^{+}(t-u) g(u) d u, \\
N(t)=\int_{0}^{1} n^{+}(t-u) \bar{G}(u) d u, \quad t \geqslant 1,
\end{gathered}
$$

where $\bar{G}$ is the tail function of the interdivision time distribution, i.e., $\bar{G}(t)=\int_{t}^{\infty} g(\tau) d \tau$.

The third hypothesis makes $m(t)$ depend on the expected cell count $N(t)$. It would be preferable to use actual cell count $\mathscr{N}(t)$ as the regulating factor. However, proceeding as we do, we obtain self-contained equations for the expected values. This would be impossible if $\mathscr{N}(t)$ were used. For more comments, see Discussion. 
HYPOTHESIS 3

The average progeny function $m(t)$ is a function of the expected cell count $N(t)$, i.e.,

$$
m(t)=\Phi[N(t)]
$$

Function $\Phi . \quad \mathbf{R}_{+} \rightarrow[0,2]$ is assumed continuously differentiable, nonincreasing, and such that $\Phi(0)>1$.

Our model assumes now its final form,

Model One.

$$
\begin{aligned}
& n^{+}(t)=\Phi[N(t)] \int_{0}^{1} n^{+}(t-u) g(u) d u, \\
& N(t)=\int_{0}^{1} n^{+}(t-u) \bar{G}(u) d u, \quad t \geqslant 0,
\end{aligned}
$$

with time variable shifted for convenience. This form of nonlinear feedback in which the total number of cells regulates the efficiency of divisions is classically known as mitotic autoregulation [23].

Let us note that Model 1 can also be derived on the basis of the partial differential equations approach (Appendix A) as suggested by Metz and Diekmann [18, Exercise III.6.2.7].

The other model is based on the following hypothesis: HYPOTHESIS 4

The average progeny function $m(t)$ is a function of the expected rate of divisions $n^{-}(t)$, i.e.,

$$
m(t)=\Phi\left[n^{-}(t)\right]
$$

Function $\Phi . \quad \mathbf{R}_{+} \rightarrow[0,2]$ is assumed continuously differentiable, nonincreasing, and such that $\Phi(0)>1$.

Solving Equations (1) and (2) for $n^{-}(t)$, and using relationship (6) we obtain a self-contained nonlinear equation for $n^{-}(t)$ :

Model Two.

$$
n^{-}(t)=\int_{0}^{1} \Phi\left[n^{-}(t-u)\right] n^{-}(t-u) g(u) d u, \quad t \geqslant 0 .
$$

In this version of mitotic autoregulation, the number of dividing cells regulates the efficiency of divisions. Equation (10) is supplemented by 
the following formula for $N(t)$ :

$$
N(t)=\int_{0}^{1} \Phi\left[n^{-}(t-u)\right] n^{-}(t-u) \bar{G}(u) d u
$$

\section{LOCAL STABILITY}

\subsection{LINEARIZED EQUATIONS}

Model 1. Let us denote $x(t)$ and $X(t)$ the solutions of the linearized problem, corresponding to $n^{+}(t)$ and $N(t)$ in system (7)-(8). We obtain

$$
\begin{gathered}
x(t)=\int_{0}^{1} x(t-u) g(u) d u-S X(t), \\
X(t)=\int_{0}^{1} x(t-u) \bar{G}(u) d u .
\end{gathered}
$$

$S$ is a nonnegative constant,

$$
S=-\frac{\Phi^{\prime}\left[\Phi^{-1}(1)\right] \Phi^{-1}(1)}{E(T)}
$$

where $\Phi^{-1}(1)$ is the equilibrium value of $N(t)$ (denoted $\bar{N}$ ), and $E(T)=\int_{0}^{1} \bar{G}(u) d u=\int_{0}^{1} u g(u) d u$ is the expected lifetime of the cell. $E(T)$ exists if Hypothesis 2 is assumed. The equilibrium value of $n^{+}(t)$ is equal to $\overline{n^{+}}=\bar{N} / E(T)$.

The characteristic equation can be obtained by substituting $x(t)=$ $a \exp (\lambda t)$ and $X(t)=A \exp (\lambda t)$, with a complex number $\lambda$, in system (12)-(13) and requiring that a solution of this form exist. This leads to a condition on the determinant of the resulting matrix:

$$
\left|\begin{array}{cc}
\int_{0}^{1}\left(1-e^{-\lambda u}\right) g(u) d u & S \\
\int_{0}^{1} e^{-\lambda u} \bar{G}(u) d u & 1
\end{array}\right|=0
$$

which can be reduced to

$$
(\lambda+S) \int_{0}^{1} e^{-\lambda u} \bar{G}(u) d u=0 .
$$


An equivalent more convenient form is obtained through integration by parts,

$$
(\lambda+S) \frac{1}{\lambda}\left[1-\int_{0}^{1} e^{-\lambda u} g(u) d u\right]=0
$$

The singularity at $\lambda=0$ is removable.

Model 2. Let us denote $y(t)$ the solution of the linearized problem, corresponding to $n^{-}(t)$ in Equation (10). We obtain

$$
y(t)=[1-S E(T)] \int_{0}^{1} y(t-u) g(u) d u
$$

The equilibrium value $\overline{n^{-}}=\Phi^{-1}(1)$ is formally the same as in Model 1 , with $S$ given by (14).

The characteristic equation can be obtained by substituting $y(t)=$ $A \exp (\lambda t)$, with a complex number $\lambda$, in Equation (18) and requiring that a solution of this form exist. This leads to the condition

$$
[1-S E(T)] \int_{0}^{1} e^{-\lambda u} g(u) d u-1=0
$$

\subsection{ROOTS OF THE CHARACTERISTIC EQUATIONS}

We first obtain two simple results concerning the roots of the characteristic equations (17) and (19).

PROPOSITION 1

Suppose Hypotheses 1, 2, 3, and 4 are satisfied. Then, the only common root of Equations (17) and (19) can be $\lambda=-S$.

Proof. Suppose that Equation (17) is satisfied and $\lambda \neq-S$. Then $\int_{0}^{1} e^{-\lambda u} g(u) d u=1$. Substitution into the left-hand side of Equation (19) yields $-S E(T)$, which is negative. Therefore Equation (19) is not satisfied.

PROPOSITION 2

Suppose Hypotheses 1, 2, and 3 are satisfied. Then, all roots of Equation (17) have negative real parts. 

of

Proof. The root $\lambda=-S$ is negative real. Let us consider the roots

$$
\frac{1}{\lambda}\left[1-\int_{0}^{1} e^{-\lambda u} g(u) d u\right]=0
$$

Suppose first that $\Re \lambda>0$. This yields $\left|\int_{0}^{1} e^{-\lambda u} g(u) d u\right| \leqslant \int_{0}^{1} e^{-\Re i \lambda u} g(u) d u$. This latter is less than 1, so that Equation (20) cannot hold. If $\Re \lambda=0$, then $\int_{0}^{1} e^{-\lambda u} g(u) d u=\iota \int_{0}^{1} \sin (\nu u) g(u) d u+\int_{0}^{1} \cos (\nu u) g(u) d u$, where $\iota$ $=\sqrt{-1}$ and $\nu=\Im \lambda$. If Equation $(20)$ is to be satisfied, then the imaginary part of the integral must be equal to 0 and the real part equal to 1 . This is possible only if $\nu=0$. This yields $\lambda=0$, which is excluded since it does not satisfy Equation (16). This concludes the proof.

Proposition 2 has the following corollary:

COROLLARY 1

If $\Phi(N)$ is continuously differentiable for $N$ near $\bar{N}$, then each trajectory $N(T)$ of Model 1, Equ ations (7) and (8), starting in some neighborhood of $\bar{N}$, approaches $\bar{N}$ as $t \rightarrow \infty$.

Model 2 was investigated repeatedly by Swick [20,21]. We quote below, in our notation, one of his results. It is assumed that the characteristic equation (19) has a dominant root, which is positive if $S E(T)<0$ and negative if $0<S E(T)<1$, and that there is $r_{0}$ such that the dominant root of the characteristic equation (19) is complex and has negative real part if $1<S E(T)<r_{0}$ and positive real part if $S E(T)>r_{0}$ The following classification of solutions of Model 2 is obtained by:

PROPOSITION 3 [20, THEOREM 1]

If $\Phi\left(n^{-}\right)$is continuously differentiable for $n^{-}$near $\overline{n^{-}}$, then the solutions of Model 2, Equation (10), satisfy the following properties:

(i) If $0<S E(T)<1$ then each trajectory $n^{-}(t)$ of Model 2, Equation (10), starting in some neighborhood of the equilibrium $\overline{n^{-}}$, approaches $\overline{n^{-}}$ with either no oscillation about $\overline{n^{-}}$or with some initial oscillations about $\overline{n^{-}}$ induced by the initial functions $n_{\mid\{-1,0\}}^{-}$

(ii) If $1<S E(T)<r_{0}$ then each trajectory of Model 2 starting in some neighborhood of $\overline{n^{-}}$approaches $\overline{n^{-}}$and oscillates about $\overline{n^{-}}$with period near $2 \mu$.

(iii) If $1<S E(T)<r_{0}$ then $\overline{n^{-}}$is unstable.

Swick's papers [20,21] provide numerous examples of equations of the type of Model 2, exhibiting periodic and even chaotic behavior. Most of these studies are based on numerical computations; however, 
for certain special forms of the feedback function $\Phi(\cdot)$, the roots of the characteristic equations can be determined analytically.

\section{GLOBAI, STABIIITY}

Model 1. We provide a result on global asymptotic stability of system (7)-(8). We begin by outlining the ideas which lead to such a result, then we state the result. The formal proof is presented in Appendix D. The reasoning is analogous to that in [4], where a more complicated system was analyzed.

It is clear from Equations (7) and (8) that the functions $n^{+}(t)$ and $N(t)$ are nonnegative for $t \geqslant 0$ if only the initial values of $n^{+}(t)$ on the interval $(-1,0)$ are nonnegative. Then, we note that $N(t)$ is absolutely continuous if only $n^{+}(t)$ is locally integrable, i.e., if $n_{(-1,())}^{+} \in L^{1}(-1,0)$.

By differentiating Equation (8) side-by-side we obtain

$$
\begin{aligned}
\dot{N}(t) & =n^{+}(t) \bar{G}(0)-n^{+}(t-1) \bar{G}(1)-\int_{t-1}^{t} n^{+}(u) g(t-u) d u \\
& =n^{+}(t)-\int_{t-1}^{t} n^{+}(u) g(t-u) d u \\
& =\{\Phi[N(t)]-1\} \int_{t-1}^{t} n^{+}(u) g(t-u) d u .
\end{aligned}
$$

Based on the above, the derivative of $N(t)$ is positive if and only if $\Phi[N(t)]-1>0$, i.e., if and only if $N(t)<\bar{N}$, and respectively it is negative if and only if $N(t)>\bar{N}$. This implies that $N(t)$ is bounded from above (and from below, since it is nonnegative). Also $\dot{N}(t)$ cannot change sign (this would required crossing $\bar{N}$ which is impossible by continuity of $N(t)$ ). Boundedness and monotonicity yield convergence of $N(t)$ to a limit $N(\infty)$, as $t \rightarrow \infty$. This limit has to be equal to $\bar{N}$. Indeed

$$
N(t)-N(0)=\int_{0}^{t}\left(\{\Phi[N(\tau)]-1\} \int_{\tau-1}^{\tau} n^{+}(u) g(\tau-u) d u\right) d \tau .
$$

If $N(t) \rightarrow N(\infty)$, then this implies that

$$
\{\Phi[N(\cdot)]-1\} \int_{-1}^{\cdot} n^{+}(u) g(\cdot-u) d u \in L^{1}[0, \infty),
$$

and so, by uniform continuity of $N(t)$, it converges to 0 , as $t \rightarrow \infty$. If $N(\infty) \neq \bar{N}$, then $\Phi[N(t)]-1$ converges to a nonzero value and so $\int_{t-1}^{t} n^{+}(u) g(t-u) d u$ converges to 0 . By Equation (7), it yields $n^{+}(t) \rightarrow$ 
0 , which by Equation (8) yields $N(t) \rightarrow 0$, a contradiction since $N(t)$ is increasing if $N(t)<\bar{N}$.

This is sufficient to demonstrate that $N(t) \rightarrow \bar{N}$ as $t \rightarrow \infty$.

PROPOSITION 4

Suppose that $n_{0}^{+} \geqslant 0$ is a nonnegative function in the space $L^{1}(-1,0)$. Then a solution of system (7)-(8), $n^{+}(t), t>-1, N(t), t \geqslant 0$, such that $n_{\mid(-1,0)}^{+}=n_{0}^{+}$, exists and both $n^{+}(t)$ and $N(t)$ are nonnegative. Moreover, $n^{+}(t)$ is locally integrable and $N(t)$ is absolutely continuous. All nonnegative solutions terld to the limit

$$
\begin{gathered}
\lim _{t \rightarrow \infty} n_{t}^{+} \stackrel{L^{1}(-1,0)}{-N} / E(T), \\
\lim _{t \rightarrow \infty} N(t)=\bar{N} .
\end{gathered}
$$

The notation $n_{t}^{+}$denotes a segment of solution $n^{+}$, understood as an element of space $L^{1}(-1,0)$, i.e., $n_{t}^{+}(s)=n^{+}(t+s)$, for $s \in(-1,0), t \geqslant 0$.

Demonstrating that $n_{t}^{+} \stackrel{L^{1}(-1,0)}{\longrightarrow} \bar{N} / E(T)$, as $t \rightarrow \infty$, constitutes the difficult part of the proof. It requires arguments similar to those in the proof of Theorem 3.3 in [4] (Appendix D).

Model 2. Swick [20] provides the following result, presented here in our notation:

PROPOSITION 5 [20, THEOREM 4]

If

$$
\begin{gathered}
\Phi\left(n^{-}\right) \geqslant a>0, \\
0<S E(T)<2, \\
\Phi\left(n^{-}\right)<1+S E(T)-\frac{S E(T)}{\overline{n^{-}}} n^{-},
\end{gathered}
$$

for $0<n^{-}<\overline{n^{-}}$, and

$$
\Phi\left(n^{-}\right)>1+S E(T)-\frac{S E(T)}{\overline{n^{-}}} n^{-}
$$

for $n^{-}>\overline{n^{-}}$, then every solution $n^{-}(t)$ of Equation $(10)$ satisfies $n^{-}(t)$ $\rightarrow \overline{n^{-}}$, as $t \rightarrow \infty$. 


\section{DISCUSSION}

Interest in dynamics of models like our Models 1 and 2 is justified since these models provide the simplest mathematical description of self-limiting growth. One classical application is mitotic autoregulation in cell populations $[4,7,23]$.

There is a substantial difference between Models 1 and 2 with respect to local and global stability. Model 1 is always asymptotically stable, while Model 2 may exhibit oscillations, period doublings, and chaos. This phenomenon is very much like what happens in the discretized logistic equation which produces complex behavior, while the basic and probably more justified continuous version does not. In our case the difference may be attributed to the smoothing influence of $N(t)$.

An equation formally identical to Equation (10) of Model 2 is considered in the book by Metz and Diekmann [18]. It can be traced back to the papers by Swick [20,21]. In these sources, it is used in a demographic context, in a way which is justified by the very specific hypotheses employed. The detailed derivation of the mathematical model used in these papers is provided by Frauenthal [9]. In the original notation, the equation has the form

$$
B(t)=\int_{\alpha}^{\beta} \phi(s) B(t-s) M\{B(t-s)\} d s, \quad t>\beta,
$$

where $B(t) d t$ is the number of female births that occur between times $t$ and $t+d t, \phi(\cdot)$ is the so-called net fertility function, and the decreasing function $M(\cdot)$ is the regulating factor which depends on past values of $B(\cdot)$. This formulation depends on the so-called Easterlin hypothesis which states that as the size of the cohort in which a female is born increases, the fertility rate for the females decreases. In [9] actual demographic data which suggest periodic behavior for $B(\cdot)$ are analyzed.

In the context of cell populations, the Easterlin-type equation

$$
n^{-}(t)=\int_{0}^{1} \Phi\left[n^{-}(t-u)\right] n^{-}(t-u) g(u) d u
$$

governs the dynamics of a model of mitotic autoregulation in which the number of dividing cells regulates the efficiency of divisions. Such a model might be true in some situations, although we do not know of any example.

It is generally recognized that stochastic and deterministic models including nonlinearities are seldom equivalent in the sense of the 
expected value of the stochastic process being equal to the deterministic process. In Equations (1)-(3) leading to Models 1 and $2, m(t)$ is precisely equal to the expected number of progeny cells per mother cell, and up to this point, these equations are accurate. The reason for this is that Equations (1)-(3) describe a linear model with variable coefficients, fully equivalent to a branching process in varying environment. Models of this type were explored by Kimmel in $[12,13]$.

Hypothesis $3, m(t)=\Phi[N(t)]$, makes $m(t)$ depend on the expected cell count $N(t)$, instead of the actual cell count $\mathcal{N}(t)$. Obviously this approximation holds only if $N(t)$ is large since only then $N(t) \sim \mathcal{M}(t)$ in some sense. This illustrates another point: Models like Models 1 and 2 make sense only if their trajectories are bounded away from 0 . Large oscillations drive the model toward regions where random fluctuations play a major role, leading to extinctions or related effects.

It is interesting to note that in two cases Model 1 can be reduced to a single equation identical to the equation of Model 2. The first case is the elementary model of mitotic autoregulation, in which cell lifetimes are exponentially distributed. Models 1 and 2 are governed by the same equation (Appendix C) and are globally asymptotically stable. This latter follows from the fact that in this particular case the dynamics is described by the ordinary differential equation,

$$
\dot{N}(t)=\{\Phi[N(t)]-1\} \alpha N(t) \text {. }
$$

It is interesting that in the general case of Model 1, the proof of global asymptotic stability includes derivation of a differential equation (21) which generalizes (23).

Another case in which both models are governed by the same equation is $m(t)=$ const (Appendix B).

Finally, let us mention the general question of equivalence of different models of population dynamics. In our recent paper [6], structured population models based on partial differential equations are compared to those stemming from branching processes. In that context, even with $m(t)=$ const, the two types of models may not be completely equivalent.

\section{APPENDIX A: THE PDE DERIVATION OF MODEL 1}

We begin with the partial differential equation, very much like, e.g., Equation (III.6.2.9) in [18]. Suppose $n(a, t)$ is the age density of cells in the population at time $t$; we write,

$$
\frac{\partial n(a, t)}{\partial t}+\frac{\partial n(a, t)}{\partial a}=-h(a) n(a, t), \quad a, t \geqslant 0,
$$


where $h(a)=g(a) / \bar{G}(a)$ is the death hazard function. This equation has the initial condition

$$
n(a, 0)=n_{0}(a)=N(0) \varphi(a), \quad a \geqslant 0,
$$

where functions $\varphi(a)$, the age density of a randomly selected member of initial population, and $f(t)$, the remaining lifetime of a randomly selected member of initial population, are related by

$$
f(t)=\int_{0}^{\infty} \varphi(a) \frac{g(t+a)}{\bar{G}(a)} d a, \quad t \geqslant 0
$$

The boundary condition,

$$
n(0, t)=n^{+}(t)=n^{-}(t) m(t), \quad t \geqslant 0,
$$

is identical to Equation (2).

Integration of the partial differential equation (24) endowed with the above initial and boundary conditions (using, for example, the method of characteristics), results in

$$
n(a, t)= \begin{cases}n_{0}(a-t) \exp \left[-\int_{a-t}^{a} h(u) d u\right] ; & t<a, \\ n(0, t-a) \exp \left[-\int_{0}^{a} h(u) d u\right] ; & t \geqslant a,\end{cases}
$$

The flux of dividing parent cells, $n^{-}(t)$, is related to the age distribution density, $n(a, t)$, by

$$
n^{-}(t)=\int_{0}^{\infty} h(a) n(a, t) d a, \quad t \geqslant 0 .
$$

Substituting the solution (28) into Equation (29), using the facts $g(a)=$ $h(a) \exp \left[-\int_{0}^{a} h(u) d u\right]$ and $\bar{G}(a)=\exp \left[-\int_{0}^{a} h(u) d u\right]$, and employing Equation (26) yield Equation (1).

APPENDIX B: THE CASE $m(t)=$ const

Let us substitute $m=$ const in system (1)-(3). Iterating Equations (1)-(3), we obtain

$$
n^{-}(t)=\int_{0}^{t} n^{-}(t-u) m g(u) d u+N(0) f(t)
$$


and

$$
n^{+}(t)=m \int_{0}^{t} n^{+}(t-u) g(u) d u+m N(0) f(t)
$$

Substitution in Equation (3) provides

$$
\begin{aligned}
N(t) & -N(0) \\
& =m \int_{0}^{t} \int_{0}^{u}\left[n^{+}(u-v)-n^{-}(u-v)\right] g(v) d v d u+(m-1) N(0) F(t) \\
& =m \int_{0}^{t} g(v)[N(t-v)-N(0)] d v+(m-1) N(0) F(t)
\end{aligned}
$$

so that

$$
N(t)=m \int_{0}^{t} N(t-B) g(v) d v+[m \bar{G}(t)-(m-1) \vec{F}(t)] N(0)
$$

If Hypothesis 2 is assumed, we see that Equation (32) is identical to Equation (9).

If the population is started by a single cell $(N(0)=1)$ born at $t=0$ (i.e., $f(\cdot)=g(\cdot)$ ), we obtain (without Hypothesis 2)

$$
N(t)=m \int_{0}^{t} N(t-v) g(v) d v+\bar{G}(t)
$$

which is the familiar renewal-type equation for the expected particle count in the Bellman-Harris branching process [8]. In the general case, Equation (32) is the equation for the expected particle count in the Bellman-Harris branching process with immigration [8].

With $t>1$, we see that Equations (30), (31), and (33) are identical.

\section{APPENDIX C: EXPONENTIALLY \\ DISTRIBUTED LIFELENGTHS}

Let us consider $g(t)=\alpha e^{-\alpha t}$. We show that in this case solutions of system (4)-(5) if they exist satisfy the equation identical to Equation (10) (with the obvious corrections of upper integration bounds).

Equation (1) is replaced by

$$
n^{-}(t)=\alpha N(t) .
$$


Indeed, substituting $g(u)=\alpha e^{-\alpha t}$ in Equation (1) yields

$$
n^{-}(t)=\int_{0}^{t} n^{+}(t-u) \alpha e^{-\alpha u} d u+N(0) \alpha e^{-\alpha t},
$$

where we set $f(t)=\alpha e^{-\alpha t}$ because, for exponentially distributed lifetimes, the remaining lifetime conditional on reaching any given age is also exponentially distributed with the same parameter. Substituting the above into Equation (3) and carrying out integrations provides

$$
\begin{array}{r}
N(t)=N(0) e^{-\alpha t}+\int_{0}^{t} n^{+}(\tau) d \tau+\int_{0}^{t} \int_{0}^{\tau} n^{+}(\tau-u) \alpha e^{-\alpha u} d u d \tau, \\
t \geqslant 0 .
\end{array}
$$

This latter yields, after a change in the integration order and a substitution,

$$
N(t)=N(0) e^{-\alpha t}+\int_{0}^{t} n^{+}(t-u) e^{-\alpha t} d u .
$$

Comparison of Equations (35) and (37) yields Equation (34).

Combining Equation (34) with Equations (2) and (3) yields the following ordinary differential equation

$$
\dot{N}(t)=\alpha[m(t)-1] N(t), \quad t \in \mathbf{R},
$$

which admits explicit solution

$$
N(t)=N(0) \exp \left\{\alpha \int_{0}^{t}[m(u)-1] d u\right\}, \quad t \in \mathbf{R},
$$

If we verify that $N(t)$ given by Equation (39) satisfies

$$
N(t)=\int_{0}^{\infty} N(t-u) m(t-u) \alpha e^{-\alpha u} d u,
$$

which leads to the desired result, then the demonstration is concluded. For this, we need an additional hypothesis.

HYPOTHESIS 5

For any $t \geqslant 0$, the integral $\int_{-\infty}^{t} m(s) d s$ is finite.

Using the variation of constants formula in Equation (38) we obtain

$$
N(t)=N\left(t_{0}\right) e^{-\alpha\left(t-t_{0}\right)}+\int_{0}^{t-t_{0}} N(t-u) m(t-u) \alpha e^{-\alpha u} d u,
$$


for any real $t$ and $t_{v}$ such that $t \geqslant t_{0}$. All the terms in Equation (41) are nonnegative, and, therefore, by passing with $t_{0}$ to $-\infty$, we obtain that the integral

$$
\int_{0}^{\infty} N(t-u) m(t-u) \alpha e^{-\alpha u} d u
$$

is finite. If Hypothesis 5 is satisfied, then convergence of this integral requires that

$$
N\left(t_{0}\right) e^{-\alpha\left(t-t_{0}\right)}
$$

tends to 0 as $t_{0} \rightarrow-\infty$. Using this in Equation (41) yiclds Equation (40), as required.

\section{APPENDIX D: PROOF OF PROPOSITION 4}

We use the standard techniques of functional analysis and theory of semigroups of operators. A review of the theory can be found in the survey by Arino [1] or in the book edited by Nagel [19].

1. The Limit Equation. Let us consider $\{T(t), t \geqslant 0\}$ the strongly continuous semigroup of bounded linear operators defined by the solutions of the equation

$$
n^{+}(t)=\int_{0}^{1} n^{+}(t-u) g(u) d u, \quad t \geqslant 0,
$$

the limit equation obtained from Equation 7 by setting $N(t) \rightarrow \infty$.

$$
T(t): L^{1}(-1,0) \rightarrow L^{1}(-1,0), \quad T(t) n_{0}=n_{t} .
$$

Since the density $g$ is essentially bounded, we have $T(t)\left(L^{1}\right) \subset L^{\infty}$ for $t \geqslant 1$.

If $g$ is in the $C^{1}$ space, then $T(1)$ sends the unit ball of $L^{\infty}$ into a bounded equicontinuous subset of $C$ and therefore $T(1)$ is compact. The essentially bounded $g$ can be approximated in the $L^{1}$ norm by a sequence of $C^{1}$ functions $g_{n}$. If we denote $T_{n}$ the semigroup associated with $g_{n}$, then $\left\|T_{n}(t)-T(t)\right\| \rightarrow 0$ as $n \rightarrow \infty$. Therefore $T(2)=$ $\lim _{n \rightarrow \infty} T_{n}(1) \circ T(1)$ is compact. This yields $T(t)$ compact for any $t \geqslant 2$.

The semigroup $T(t)$ has a characteristic equation

$$
1=\int_{0}^{1} e^{-\lambda u} g(u) d u \text {. }
$$


Equation (44) has only roots with nonpositive real parts. Moreover, in view of $\int_{0}^{1} g(u) d u=1$ and $g \geqslant 0$, we conclude that $\lambda=0$ is the only root with zero real part.

Since $T(t)$ is a translation semigroup, its infinitesimal generator is defined by

$$
\begin{gathered}
A \varphi=\dot{\varphi} \\
D(A)=\left\{\varphi \in C^{1}([-1,0]): \varphi(0)=\int_{0}^{1} \varphi(-u) g(u) d u\right\} .
\end{gathered}
$$

The algebraic multiplicity of $\lambda=0$ is 1 , but this is not sufficient for the dimension of its generalized eigenspace to be 1 . However, suppose that this dimension is $\geqslant 2$. Then, there exists a $u \in D(A) \backslash \operatorname{Ker}(A-\lambda \mathrm{Id})$ such that $(A-\lambda \mathrm{Id})^{2} \varphi=0(\lambda=0)$. This latter implies $\varphi^{\prime \prime}=0$ and $\varphi=$ $C_{1} u+C_{2}$. Requirement $\varphi \in D(A)$ yields the condition $0=-C_{1} E(T)$, which implies $C_{1}=0$, i.e., $\varphi=C_{2} \in \operatorname{Ker}(A-\lambda \mathrm{Id})$, which is a contradiction.

2. The nonlinear Problem. We know that $N(t) \rightarrow \bar{N}$ and that $N(t)$ is absolutely continuous and so bounded. Departing from this, we prove that $n^{+}$is bounded from above. There exists $\epsilon>0$ such that $\bar{G}(\epsilon)=1 / 2$. This implies, by Equation (8),

$$
\frac{1}{2} \int_{0}^{\epsilon} n^{+}(t-u) d u \leqslant \max _{\tau \geqslant 0} N(\tau) .
$$

But if this is true for each $t \geqslant 0$, then also $\int_{0}^{1} n^{+}(t-u) d u \leqslant M_{1}<\infty$, and from this, using Equation (7), $n^{+}(t) \leqslant M_{2}<\infty$.

Using the same technique as for the linear case, we demonstrate that the trajectory of any solution of the nonlinear equation is relatively compact. Let us consider a subsequence $n_{t_{n}}^{+}$which converges to $\bar{n}$ as $t_{n} \rightarrow \infty$. The limit $\tilde{n}$ is a solution of the limit equation (42) on the whole real axis. Moreover, $\tilde{n}$ must satisfy the condition

$$
\bar{N}=\int_{0}^{1} \tilde{n}(t-u) \bar{G}(u) d u .
$$

Since $\tilde{n}$ is a solution of the limit equation, we have (see the discussion in Part 1 of the proof) $\tilde{n} \stackrel{L_{1}}{\longrightarrow} \overline{n^{+}}$, as $t \rightarrow \infty$, where $\overline{n^{+}}-\bar{N} / E(T)$.

We prove that $\tilde{n}=\bar{n}^{+}$. Because the spectrum of an eventually compact semigroup is a pure point spectrum (except perhaps the spectral element at 0 ), and since $e^{0 t}=1$ is the strictly dominant cigenvalue of $T(t)$, the convergence of $\tilde{n}$ toward $n^{+}$is exponential with, say, exponent 
$-\gamma, \gamma>0$. Therefore, if $t<0$, we have

$$
\left\|\tilde{n}_{0}-\overline{n^{+}}\right\| \leqslant M\left\|\tilde{n}_{t}-\overline{n^{+}}\right\| e^{-y|l|}, \quad M \geqslant 1 .
$$

Therefore, if $\tilde{n} \neq \overline{n^{+}}$, we conclude that $\left\|\tilde{n}_{t}-\overline{n^{+}}\right\| \rightarrow \infty$, as $t \rightarrow-\infty$. This is impossible since $\tilde{n}$ is bounded on $\mathbf{R}$.

The above implies $\tilde{n}=\overline{n^{+}}$. Since this is true for each element of the $\omega$ - limit set of $n^{+}$, this set is reduced to a single element; i.e., the solution tends to a constant.

The first version of this paper was written in Spring 1993, when Ovide Arino visited the Department of Statistics, Rice University. Ovide Arino was supported in part by a grant from the French National Center for Research, program Environment, entitled Environment and Structured Populations. Marek Kimmel was supported in part by grants (DMS 9020626 and DMS 9203436) from the National Science Foundation.

\section{REFERENCES}

1 O. Arino, Some spectral properties for the asymptotic behavior of semigroups connected to population dynamics, SIAM Rev. 34:445-476 (1992).

2 O. Arino, D. E. Axelrod, and M. Kimmel, eds., Mathematical Population Dynamics, Lecture Notes in Pure and Applied Mathematics, Dekker, New York, (1991).

3 O. Arino, and M. Kimmel, Asymptotic analysis of a cell cycle model based on unequal division, SLAM J. Appl. Math. 47:128-145 (1987).

4 O. Arino, and M. Kimmel, Asymptotic behavior of a nonlinear functional-integral equation of cell kinetics with unequal division. J. Math. Biol. 27:341-354 (1989).

5 O. Arino, and M. Kimmel, Asymptotic behavior of nonlinear semigroup describing a model of selective growth regulation, J. Math. Biol. 29: 289-314 (1991).

6 O. Arino, and M. Kimmel, Comparison of approaches to modeling of cell population dynamics, SIAM J. Appl. Math. 53:1480-1504 (1993).

7 O. Arino, M. Kimmel, and M. Zerner, Analysis of a cell population model with unequal division and random transition, in Mathematical Population Dynamics (O. Arino, D. E. Axelrod, and M. Kimmel, eds.), Lecture Notes in Pure and Applied Mathematics, Dekker, New York, 1991, pp. 3-12.

8 K. B. Athreya and P. E. Ney, Branching Processes, Springer, New York, 1972.

9 J. C. Frauenthal and K. E. Swick, Limit cycle oscillations of the human populations, Demography 20:285-298 (1983).

10 M. Kimmel, Cellular population dynamics. I. Model construction and reformulation, Math. Biosci. 48:211-224 (1980).

11 M. Kimmel, Cellular population dynamics. II. Investigation of solutions, Math. Biosci. 48:225-239 (1980).

12 M. Kimmel, Equivalence result for integral equations with applications to branching processes, Bull. Math. Biol. 44:1-15 (1981). 
13 M. Kimmel, The point process approach to age- and time-dependent branching processes, Adv. Appl. Probab. 15:1-20 (1993).

14 M. Kimmel, Metabolic events in the cell cycle of malignant and normal cells, in Cancer Modeling, (J. R. Thompson and B. W. Brown, eds., Dekker, New York, 1987, pp. 215-235.

15 M. Kimmel, and O. Arino, Cell cycle kinetics with supramitotic control, two cell cycles, and unequal division: A model of transformed embryonic cells, Math. Biosci. 105:47-79 (1991)

16 M. Kimmel and D. E. Axelrod, Mathematical model of the dynamics of unequal division, growth regulation and colony size of mammalian cells, J. Theor. Biol. 153:157-180 (1991).

17 M. Kimmel, Z. Darzynkiewicz, O. Arino, and F. Traganos, Analysis of a cell cycle model based on unequal division of metabolic constituents to daughter cells during cytokinesis, J. Theor. Biol. 110:637-664 (1984).

18 J. A. J. Metz, and O. Diekmann, The Dynamics of Physiologically Structures Populations, Lecture Notes in Biomathematics, Vol. 68, Springer, New York, 1986.

19 R. Nagel, Editor One-parameter Semigroups of Positive Operators, Lecture Notes in Mathematics, vol. 1184, Springer, New York, 1986.

20 K. E. Swick, Stability and bifurcation in age-dependent population dynamics, Thear. Pop. Biol. 20:80-100 (1981).

21 K. E. Swick, Some reducible models of age dependent dynamics, SIAM J. Appl. Math. 45:256-267 (1985).

22 G. F. Webb, Random transitions, size control, and inheritance in cell population dynamics, Math. Biosci. 85:71-91 (1987).

23 T. E. Wheldon, Mathematical Models in Cancer Research, Adam Hilger, Philadelphia, 1988. 